\title{
Compétence paraphrastique et interprétation : Le verbe et ses entours en grande section de maternelle ( 5 ans)
}

\author{
Anne Sardier \\ Univ. Limoges, CeReS, EA 3648, F-87000 Limoges, France.
}

\begin{abstract}
Résumé. L'enseignement du lexique est un incontournable des acquisitions à l'école maternelle. Il fait l'objet d'ateliers dits 'de langage"' auprès des jeunes élèves. C'est alors souvent un lexique nominal et concret qui leur est enseigné via des cartes-images. Le verbe, pivot de la phrase, est ainsi peu étudié (excepté certains verbes d'action). C'est la raison pour laquelle nous avons souhaité travailler sur ce matériau lexical. Par ailleurs, il s'avère que la dimension syntagmatique du lexique, si elle participe à la construction sémantique de l'unité lexicale, est également peu mobilisée dans l'enseignement. La compétence lexicale se construisant au vu de l'accroissement du lexique disponible, mais aussi au vu des habiletés des élèves et des stratégies qu'ils peuvent mettre en place, nous supposons que le fait d'enseigner des stratégies de précision du sens des verbes va les aider à s'approprier ce lexique. À partir de l'analyse de verbes sélectionnés avec deux enseignants de grande section (5 ans), le présent article présente un dispositif d'exploration des verbes et de leur entour via la paraphrase, habileté essentielle dans les acquisitions lexicales, afin de favoriser la compréhension et le réemploi des verbes étudiés. Trois tests émaillent la mise en œuvre, les séances ont été enregistrées et retranscrites. Leurs analyses tendent à montrer que les élèves comprennent mieux les verbes étudiés et qu'ils se les sont appropriés au point de pouvoir les réemployer.
\end{abstract}

Mots clés : lexique, paraphrase, acquisition

\begin{abstract}
Teaching lexicon is important for acquisition at kindergarten. It is the subject of language workshops with young students. It is then often a nominal and concrete lexicon, which is taught to them with flashcards. The verb, pivot of the french sentence, is thus little studied (except some action verbs). This is the reason why we wanted to work on this lexical material. Furthermore, it turns out that the syntagmatic dimension of the lexicon, if it participates to the semantic construction of lexical unity, is also little used in teaching. The lexical competence being built by the increase in the lexicon available, but also by the skill of the students and the strategies they can implement, we suppose that the fact of teaching strategies of precision of the meaning of verbs will help them to appropriate this lexicon. Based on the analysis of verbs selected with two teachers of kindergarten (5 years), this article presents a device for exploring verbs and their surrounds via paraphrase, an essential skill in lexical acquisitions, in order to promote understanding and reuse of the verbs studied. Three tests punctuate the implementation, the sessions were recorded and transcribed. Their analyzes tend to show that the students understand the verbs studied better and that they appropriated this verbs to the point where they can be used again.
\end{abstract}

Keywords: lexicon, paraphrase, acquisition

\section{Introduction}

Mobiliser l'analyse sémantique des verbes dans une visée praxéologique pour favoriser les apprentissages lexicaux de jeunes enfants, est-ce judicieux? À la suite d'Aurnague \& Garcia-Debanc (2016), nous nous 
proposons d'apporter des éléments de réponse à cette question à partir de l'analyse de verbes choisis par des enseignants de grande section ( 5 ans). L'enseignement du lexique est en effet un incontournable de l'école maternelle. Il fait l'objet d'ateliers dits "de langage'" auprès des jeunes élèves. C'est alors souvent un lexique nominal ou concret qui leur est enseigné via des cartes-images. Le lexique verbal est peu étudié, excepté certains verbes d'action comme les verbes de déplacement par exemple (Garcia-Debanc et al., 2009 ; Roubaud \& Moussu, 2014), il souffre encore «d'une grande disette » (Picoche, 1999, p. 3). Pourtant le verbe est largement mobilisé dans les diverses activités de l'école. C'est la raison pour laquelle nous avons souhaité travailler sur ce matériau lexical. Par ailleurs, il s'avère que la dimension syntagmatique du lexique, si elle participe à la construction sémantique de l'unité lexicale, est également peu mobilisée dans l'enseignement (Chanfrault-Duchet, 2004 ; Sardier, 2016b). Or, compte tenu du fait que la compétence lexicale se construit au vu de l'accroissement du lexique disponible, mais aussi au vu des habiletés des élèves (Tremblay, 2009) et des stratégies qu'ils peuvent mettre en place (Sardier, 2016a), nous supposons que le fait d'enseigner des stratégies de précision du sens des verbes va aider les élèves à s'approprier ce lexique. Nous avons alors sélectionné une liste de verbes que les enseignants souhaitaient travailler avec leurs élèves. Afin d'expliquer notre démarche, nous revenons ci-dessous dans un premier temps sur la sélection et l'analyse de ce matériau lexical, et sur l'enseignement du lexique en maternelle. Nous expliquons dans un deuxième temps la démarche adoptée dans le cadre de cette étude et nous apportons des précisions sur le déroulement de la proposition didactique faite aux enseignants pour leurs élèves. Enfin, il s'agira dans un troisième temps d'analyser les effets de cette mise en œuvre sur les apprentissages lexicaux à partir des enregistrements des séances de classe. Nous allons alors chercher à savoir 1) si les élèves comprennent mieux les verbes étudiés et 2) s'ils se les sont appropriés au point de pouvoir les réemployer.

\section{Comment et pourquoi explorer une unité lexicale?}

\subsection{Interactions au sein du système lexical}

« Déplier la notion » (Nonnon, 2008, p. 64), « décortiquer les unités lexicales » (Polguère \& Tremblay, 2003 , p. 27), tels sont quelques-uns des termes utilisés par les chercheurs pour qualifier les activités mises en œuvre dans le cadre de la didactique du lexique. Il s'agit « de " tirer » sur une lexie de ce réseau [lexical] pour que vienne avec elle toute une série d'autres lexies auxquelles elle semble attachée par des liens parfois très subtils» (Polguère, 2002, p. 80). Ce sont précisément ces « liens subtils » que nous souhaitons permettre aux tout jeunes élèves d'appréhender car ils permettent d'une part de mieux comprendre les unités étudiées et, d'autre part, de mieux comprendre comment fonctionne le système lexical. D'un point de vue linguistique, trois axes guident là notre réflexion.

D'abord, une lexie entretenant avec d'autres des relations de sens, de forme et de combinatoire, afin de déplier un mot, il s'agit de considérer le fonctionnement de ces trois types de relations. Pour parvenir à analyser ce fonctionnement, la polysémie est une entrée productive en didactique du lexique parce qu'elle force "à isoler les différentes acceptions d'un mot pour pouvoir les caractériser et les différencier » (Polguère \& Tremblay, 2003, p. 28). Or, la sélection d'un sens pertinent s'effectue par rapport aux autres unités de la phrase, et le choix d'une acception est possible à partir du cotexte de l'unité lexicale.

Ensuite, nous entendons par cotexte les cooccurrents fréquents d'une unitéi à l'échelle de la phrase, ils vont déterminer l'actualisation d'une lexie. Les dimensions sémantique et syntagmatique du lexique se trouvent ainsi étroitement liées. Par exemple pour briser, que nous étudierons plus bas, les cooccurrents verre, triste, ou cour dans des phrases telles «Sylvia a brisé un verre » ou bien « La reine des Neiges est triste car le prince lui a brisé le cœur» entrainent des variations sémantiques de l'unité briser et permettent d'en préciser le sens. La mobilisation des cooccurrents du verbe permet :

de construire son champ lexical à partir du sens des cooccurrents qui influent sur celui de l'unité de base,

de sélectionner une acception pertinente au sein du champ lexical construit. 
Le cotexte entretient ainsi une relation d'ordre dynamique avec l'unité : il permet de construire le champ de ses variations sémantiques, mais il déclenche aussi l'activation du sens dans l'énoncé.

Enfin, ajoutons que « les verbes, du fait de leur fréquence et de la difficulté de leurs constructions syntaxiques, doivent faire l'objet d'une attention particulière (Garcia-Debanc et al. 2009, p. 214). Le verbe étant en outre le pivot de la phrase française, son étude implique celle de ses entours (sujet et compléments) qui seront autant de « places vides, qu'il faut remplir par des noms » (Picoche, 2011, p. 4). Le cotexte représenté par des noms est par ailleurs souvent déjà connu des élèves (Bassano, 2005, 2010) et il est donc possible d'y recourir pour l'interprétation. Les interactions au sein du système impliquent ainsi de considérer simultanément lexique et grammaire.

Quand c'est possible, en utilisant les éléments cotextuels, l'apprenant se penche sur l'unité elle-même et son champ lexical, dans ses relations avec les autres unités. Notons cependant que cette influence mutuelle joue essentiellement dans le cas de combinaisons libres ou semi-figées, mais elle ne peut jouer pour des expressions figées dont le sens sera non compositionnel et qui seront à comprendre et mémoriser telles quelles.

\subsection{Stratégie d'interprétation et compétence paraphrastique}

Si la compétence lexicale est affaire de connaissances et d'habiletés, alors il s'agit d'orienter l'enseignement selon ces deux axes. Les connaissances lexicales sont de deux ordres : un stock d'unités lexicales disponibles et une connaissance du fonctionnement du système (liens entre les unités et relation au contexte). Les connaissances lexicales demandent de prendre en compte le temps de la mémorisation. C'est d'ailleurs l'un des éléments déterminants du réemploi lexical (Sardier \& Grossmann, 2010) : le réemploi ne sera possible qu'à partir du moment où l'unité sera suffisamment bien comprise et mise en mémoire pour être disponible et mobilisable. Les habiletés lexicales sont liées à la capacité à mobiliser le lexique disponible ou à utiliser les relations entre les unités pour y recourir en cohérence avec la situation. Ainsi la compétence lexicale implique une représentation approfondie du lexique conjuguée à la capacité à s'y référer en fonction du contexte. Du point de vue de la didactique du lexique, nous prenons ici principalement appui sur deux concepts.

D'une part, cette imbrication entre les différentes composantes de la compétence lexicale nous incite à penser que les élèves peuvent avoir besoin d'un enseignement des stratégies leur permettant de mobiliser le lexique à bon escient. La capacité métalinguistique consistant à solliciter les cooccurrents fréquents d'une unité lexicale (son cotexte) fait partie des stratégies possibles pour produire l'inférence lexicale nécessaire à une bonne interprétation des unités (Sardier, 2018). Cependant, il apparait que la capacité à mobiliser une telle stratégie n'est pas enseignée aux tout jeunes élèves. C'est la raison pour laquelle nous la proposons en classe de grande section (GS). Les élèves sont en effet capables d'une réflexion lexicale poussée dès cet âge là (Sardier, 2016a), et nous voulons savoir dans quelle mesure l'entrainement à mobiliser le cotexte d'une unité lexicale pourra aider à l'acquisition de cette même unité.

D'autre part, le rôle de la paraphrase est ici déterminant car « il n'y a pratiquement pas d'autre façon naturelle de procéder pour décrire le sens que de faire appel à des paraphrases » (Polguère, 2002, p. 96). La compétence paraphrastique consiste alors à «reformuler des phrases en créant des énoncés sémantiquement équivalents » (Tsedryk, 2014, p. 1). En outre, « l'acquisition de la langue se réaliserait en grande partie à travers l'acquisition de la compétence paraphrastique » (Martinot, 2010, p. 63). Plusieurs types de paraphrases peuvent être distinguées (Martinot, 2010 ; Tsedryk, 2014) selon que les apprenants mobilisent :

des synonymes termes à termes (ex. : « la peur hante > obsède le jeune garçon »),

des décompositions de termes à partir de la synonymie ou de la morphologie (ex. : « les ombres hantent > marchent partout dans les corridors »; « le verre est brisé » > « il est tombé, il y a plein de brisures par terre ») 
des équivalences selon le contexte (ex. : «les ombres hantent les couloirs > il y en a partout dans l'appartement)

- des transformations syntaxiques formelles comme une permutation d'éléments (ex. : « la peur hante le jeune garçon »> « le jeune garçon est hanté par la peur »).

Les trois premiers types de paraphrases témoignent de la compréhension du sens du mot étudié puisqu'il y est remplacé, glosé, par des équivalents sémantiques. La transformation syntaxique témoigne plutôt d'une compréhension de l'organisation grammaticale, mais ne peut être considérée comme révélatrice d'une bonne appréhension du sens du verbe puisque, en l'absence de glose explicative il sera difficile de savoir si l'apprenant mobilise effectivement le sens ou non. Dans la mesure où elles assurent la continuité sémantique entre le mot étudié et ses remplaçants, les trois premiers types de paraphrases seront pris en compte dans l'analyse ci-dessous. L'objectif est alors de proposer et tester un dispositif qui puisse permettre à des enfants de 5 ans de réfléchir aux relations entre le vocable et ses lexies au moyen de reformulations paraphrastiques.

\section{Le verbe et ses entours en grande section de maternelle}

\subsection{Sélection et analyse du matériau lexical}

Les activités linguistiques proposées à l'école maternelle sont grandement orientées vers les acquisitions lexicales. Florin (2010) rappelle d'ailleurs à quel point il est déterminant de favoriser le développement du lexique à cet âge. L'accent est alors mis sur les noms en vertu du fait que les catégories grammaticales ne sont pas acquises en même temps et que les noms le sont en priorité (Bassano, 2005, 2010). Pourtant, à partir de 20 mois, la tendance s'inverse et la fréquence des verbes égale celle des noms (Bassano, 2010). Il pourrait dès lors être productif en didactique de considérer cette évolution naturelle pour la mettre à profit et enseigner davantage de verbes avec les jeunes élèves. Poursuivant une visée praxéologique, nous avons alors questionné à ce sujet deux enseignants maitres formateurs officiant à l'école maternelle pour savoir sur quels verbes ils souhaiteraient que nous engagions avec eux un travail de recherche. C'est donc à leur demande que nous avons choisi de nous pencher sur l'enseignement-apprentissage des verbes hanter, et briser en grande section de maternelle $e^{\text {ii }}$. Les enseignants remarquent en effet que les emplois métaphoriques de ces verbes entrainent nombre d'incompréhensions chez leurs élèves et ils souhaitaient pouvoir tenter de remédier à cette difficulté.

Étant donné qu'une « analyse des sens lexicaux est requise si l'on veut pouvoir identifier clairement les relations sémantiques entre lexies " (Polguère, 2002, p. 144), nous avons dans un premier temps procédé à l'analyse de ces deux verbes, avant d'envisager un dispositif didactique à mettre en place pour leur enseignement-apprentissage auprès des élèves de grande section de maternelle. Pour l'analyse nous utilisons prioritairement les articles du TLFi et les différents onglets proposés pour chaque article par le CNRTL (notamment l'onglet proxémie pour compléter la synthèse des différents liens synonymiques). Le dictionnaire de Dubois \& Dubois-Charlier (1997), qui recense trois acceptions pour hanter et neuf pour briser a également été utilisé. Dans une perspective didactique, l'analyse peut se résumer très succinctement comme suit : 
Hanter

Occuper par son omniprésence

Effrayer, tourmenter, altérer par son

omniprésence

\section{1 - L'occupation s'exerce sur un lieu}

Ex. : Les ombres hantent les corridors

Figure 1 Synthèse des sens de hanter
2 - L'occupation s'exerce sur une personne, par une émotion, un sentiment, une pensée obsédante

Ex. : La peur de revoir ces monstres hantait continuellement le jeune garçon

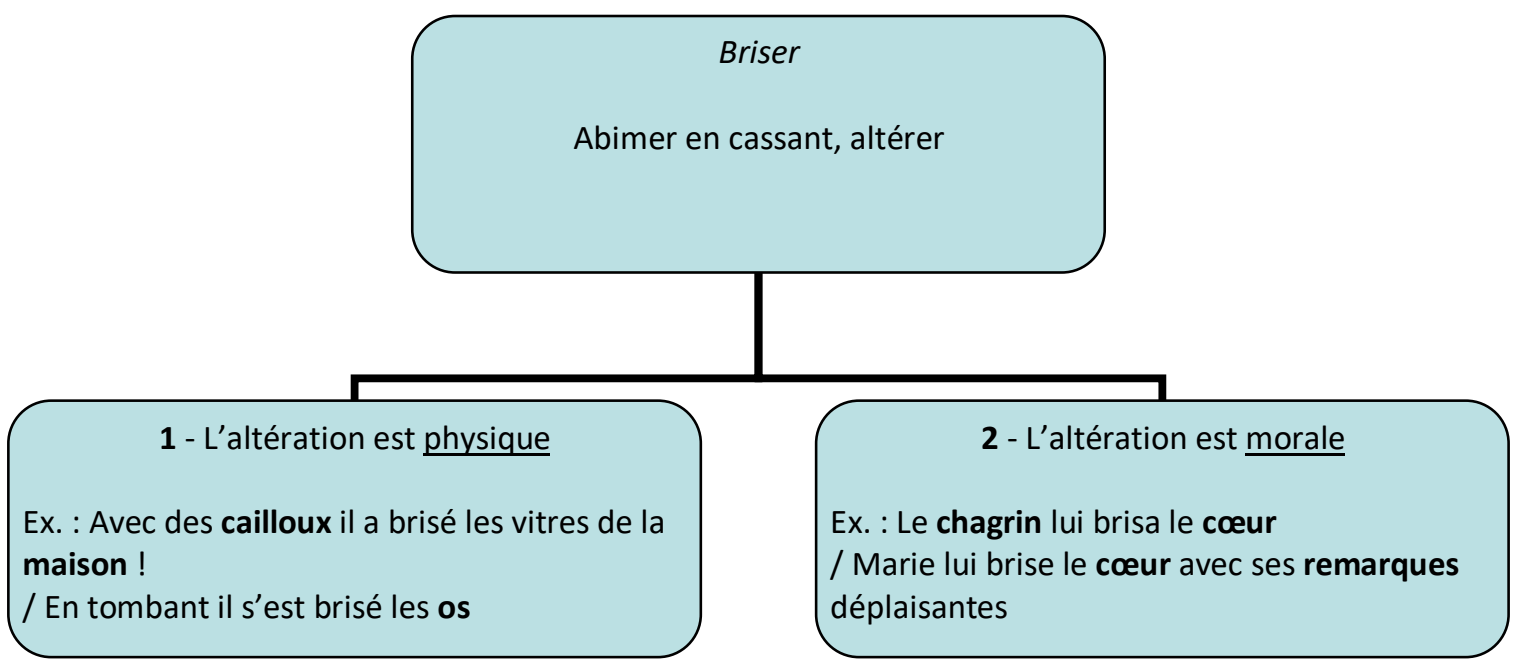

\section{Figure 2 Synthèse des sens de briser}

À l'analyse, nous constatons que ces deux verbes ont une caractéristique sémantique commune : ils sont révélateurs d'une modification négative exercée par un actant sur un autre, que ce soit sur une chose ou un lieu (sens 1) ou sur l'esprit (sens 2). Par exemple Le château est hanté par... vs La peur hante Maxime, etc. Nous pouvons dire que ce sont des verbes d'altération, et c'est en fonction de cette principale acception que nous allons nous intéresser à hanter et briser dans une perspective didactique.

De telles unités lexicales peuvent être difficiles à comprendre pour les jeunes élèves dans la mesure où d'une part elles sont employées dans un sens figuré et où, d'autre part, ils peuvent les connaitre déjà dans leur sens premier ce qui est susceptible d'engendrer une interprétation erronée de l'unité lexicale suivant le contexte d'emploi. 
Dans les deux cas, si nous nous intéressons aux " place vides à remplir » autour de ces verbes, il est possible de s'appuyer sur les sens des éléments du cotexte qui entrainent la mobilisation du sens figuré (par exemple la peur) afin d'interpréter l'unité en contexte phrastique. L'étude de la dimension syntagmatique entraine alors une exploration du champ des variations sémantiques des unités lexicales. Cette focalisation sur les sens des éléments cotextuels peut permettre d'orienter les élèves vers les éléments favorisant une désambigüisation des unités lexicales lorsque leur sens peut poser problème, pour qu'ils puissent construire l'inférence lexicale. Ce recours au cotexte constitue une stratégie d'interprétation pour de jeunes élèves : « tu écoutes bien les mots qui sont autour pour t'aider ».

De cette analyse sémantique il est possible d'extraire, dans un but didactique, une analyse syntagmatique permettant de catégoriser les unités lexicales auxquelles il faut s'intéresser pour mieux comprendre le fonctionnement du vocable. Autour du verbe, nous trouvons prioritairement des noms, mais, si nous nous intéressons à l'environnement phrastique, il est possible de trouver également d'autres verbes et des adjectifs qui vont aider à l'interprétation. Ainsi autour de hanter ou briser, nous pouvons relever des exemples des représentants de ces deux classes :

Tableau 1. Hanter et briser : catégorisation des éléments cotextuels à des fins didactiques

\begin{tabular}{|c|c|c|}
\hline & $\begin{array}{c}\text { Noms communs / propres } \\
\text { Adjectifs }\end{array}$ & Verbes \\
\hline Hanter & $\begin{array}{l}\text { Les ombres hantent les corridors } \\
\text { Pablo est hanté par la peur }\end{array}$ & $\begin{array}{l}\text { Les ombres habitent les couloirs et les } \\
\text { hantent la nuit. } \\
\text { Les fantômes qui hantent le château } \\
\text { terrorisent ses habitants }\end{array}$ \\
\hline Briser & $\begin{array}{l}\text { Il a brisé le miroir } \\
\text { Pierre est triste, le chagrin lui brise le } \\
\text { cœur. }\end{array}$ & $\begin{array}{l}\text { Le miroir s'écrasa à terre et se brisa } \\
\text { Le vase se renverse, tombe et se brise. }\end{array}$ \\
\hline
\end{tabular}

Dans le cas d'un enseignement-apprentissage auprès d'élèves de 5 ans, cette catégorisation grammaticale des éléments cotextuels constituera une base de travail pour l'enseignant qui pourra ainsi mieux étayer les échanges et guider la réflexion de ses élèves en leur permettant de prêter attention aux mots permettant l'interprétation. Ce type de structuration des " places vides » constitue alors un élément de formation pour l'enseignant afin qu'il puisse utiliser la dimension syntagmatique du lexique à des fins d'apprentissage.

Cette stratégie du recours aux unités cotextuelles favorise en effet la précision du sens par construction de l'inférence lexicale. Il peut alors être possible d'entrainer les élèves à interroger ces mots qui peuvent aider en cas d'ambigüité.

\subsection{Types de données et angles d'analyse}

Deux classes ont participé à cette étude, le dispositif a été préalablement construit, puis aménagé avec deux professeurs des écoles maitres formateurs de l'école de Canteloube à Aurillac, Myriam Sala et Bruno Frégeac. Ils ont mené les séances simultanément avec leurs élèves. Des enregistrements des échanges oraux ont systématiquement eu lieu lors des tests, ainsi que tout au long du dispositif.

L'objectif est de savoir si, grâce à des séances centrées sur les entours des verbes étudiés, les élèves ont accru leur capacité à en préciser le sens en recourant aux dimensions du système lexical. Pour évaluer 
les effets produits par le dispositif sur la compréhension du fonctionnement des unités étudiées et sur leur réemploi, nous analysons les échanges au vu de la mobilisation d'éléments relatifs aux dimensions du système, par exemple des synonymes, des cooccurrents, des mots de la même famille morphologique. Nous cherchons également les occurrences témoignant du réemploi des verbes étudiés dans les différents échanges oraux analysés ; il s'agit alors de savoir si la mise en relation des différentes facettes d'une unité lexicale en favorise l'appropriation.

Pour le prétest, afin de savoir si les élèves avaient déjà au départ une connaissance des verbes à étudier et, si oui, pour combien d'entre eux, les verbes leur ont été présentés dans de courtes phrases. Les enseignants ont demandé à chaque enfant individuellement ce que voulait dire selon eux telle ou telle phrase. Si l'élève parvenait à paraphraser (synonyme, décomposition ou équivalence) la situation, nous avons considéré qu'il témoignait de sa compréhension du fonctionnement du système et de l'unité lexicale cible ; inversement s'il ne parvenait pas à produire de paraphrase. Étant donné le choix du matériau lexical, il n'était pas possible de proposer comme déclencheur des images comme cela peut être le cas en maternelle (les images ont été sollicitées par la suite en guise de mémoire des acceptions découvertes au fur et à mesure, par exemple pour briser: une image de verre cassé, puis ensuite une image de cœur cassé ; ces images correspondaient aux cooccurrents de briser, autrement dit au cotexte). Ainsi le dispositif s'appuie effectivement sur la compétence paraphrastique des élèves.

Un second test a consisté en un jeu d'appariement entre les verbes et les images de leurs cooccurrents, appelés « mots-amis ». Les élèves devaient redire les verbes étudiés, tirer une carte représentant l'un des mots amis et dire avec quel verbe il la mettrait en expliquant. Il s'agit là de savoir là si les élèves ont compris l'interaction entre dimension sémantique et syntagmatique (c'est-à-dire s'ils ont compris comment fonctionne le système).

Un troisième test a eu lieu lors d'un temps de synthèse des acceptions étudiées. À partir des paraphrases, il s'agit ici de savoir si les élèves ont compris les différents sens des verbes et s'ils sont capables de les réemployer.

Le tableau ci-dessous synthétise les procédés d'analyse mis en œuvre pour évaluer le dispositif :

Tableau 2. Le verbe et ses entours en GS : synthèse des procédés d'analyse

\begin{tabular}{|c|c|l|}
\hline Données & Items analysés & \multicolumn{1}{c|}{ Indicateurs } \\
\hline Prétest & $\begin{array}{c}\text { Connaissances } \\
\text { préalables }\end{array}$ & Paraphrases : synonymes, décomposition, équivalence \\
\hline Test 2 & $\begin{array}{c}\text { Stratégie } \\
\text { d'interprétation }\end{array}$ & $\begin{array}{l}\text { Associations syntagmatiques : appariement verbe / cooccurrent } \\
+ \text { Gloses explicatives (paraphrases) }\end{array}$ \\
\hline Test 3 & Réemploi lexical & $\begin{array}{l}\text { Occurrences des verbes et éléments cotextuels dans les temps de } \\
\text { synthèse }\end{array}$ \\
\hline
\end{tabular}

Les trois phases de test permettent d'apporter des éléments de réponse à notre question : le recours par de jeunes apprenants aux cooccurrents des verbes et à la paraphrase permet-il de favoriser les acquisitions lexicales?

\subsection{Parcours proposé aux élèves}

Le parcours proposé aux élèves s'est déroulé sur une période scolaire ( $\approx 5$ semaines) au printemps 2019 , le réinvestissement ultérieur a eu lieu en période suivante.

Plusieurs temps d'enseignement du lexique ont eu lieu : 
- $\quad$ semaine 1 : faire émerger le lexique ou les sens disponibles (phrases à paraphraser)

- $\quad$ semaine 2 : rappeler la première activité et synthétiser les sens premiers (images : cf. annexe 1)

- $\quad$ semaine 3 : découvrir les autres acceptions et les mots-amis (nouvelles phrases)

- $\quad$ semaine 4 : rencontrer les mots (enseignement indirect)

- $\quad$ semaine 5 : manipuler les verbes avec leur cotexte (appariement, cf. annexe 2)

synthétiser l'ensemble des acceptions découvertes

semaine 6 : réinvestir ultérieurement.

Un temps dit d'imprégnation d'une semaine a été aménagé en semaine 4. Ce temps a permis aux enseignants et aux élèves de mobiliser le lexique étudié dans diverses situations quotidiennes de la classe.

Concernant les supports, les élèves disposaient d'une affiche collective (annexe 1) sur laquelle les verbes étaient écrits avec les images illustrant les premières acceptions découvertes. Ces images constituent une illustration de la paraphrase sémantique correspondant au premier sens mobilisé par les élèves.

Nous voyons que, dans ce dispositif, les verbes sont étudiés, leur cotexte est mobilisé, et que les acceptions sont expérimentées par les élèves dans le cadre de la vie de la classe.

Les objectifs didactiques poursuivis sont de trois ordres :

- accroitre le vocabulaire des élèves,

- $\quad$ structurer le vocabulaire des élèves en mettant les mots en relation (relation de sens, relation cotextuelle),

- proposer des stratégies de précision (désambigüisation) du lexique en incitant à mobiliser le cotexte des mots, en plus du contexte.

\section{Résultats : structuration et accroissement du lexique}

Les premières analyses des transcriptions suggèrent des résultats dans deux directions révélatrices des progrès des élèves.

\subsection{Une exploration approfondie des unités lexicales}

Les analyses montrent que les jeunes enfants passent d'un travail hésitant à un examen approfondi du sens des unités lexicales étudiées. Ainsi, en début de parcours, il leur est difficile d'exprimer leur compréhension des unités lexicales. 


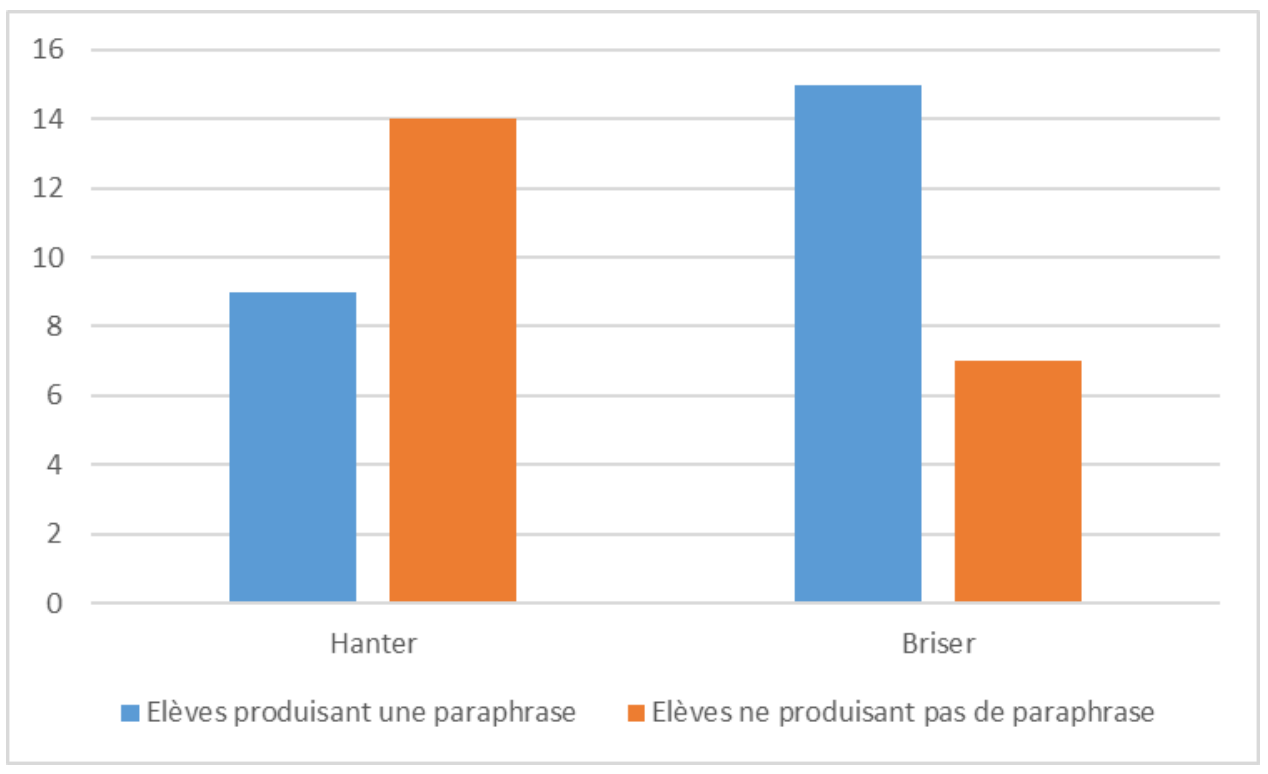

Fig. 3. En début de parcours : nb d'élèves manifestant leur compréhension des verbes étudiés $(\mathrm{N}=26)$

L'étude des connaissances préalables des élèves donne à voir leur familiarité (Babin, 2000) avec les verbes proposés. Briser, dont le sens peut être concret, est davantage paraphrasé que hanter puisqu'un peu plus de la moitié des élèves parviennent à produire une paraphrase acceptable lors du prétest. Briser est en outre souvent mis en relation avec un scénario explicatif qui peut sembler vécu par les élèves. Ce premier état des connaissances montre l'intérêt de questionner, avec les élèves, les sens métaphoriques qui sont souvent mobilisés dans les textes littéraires ${ }^{i i i}$. Concernant l'analyse de ces connaissances préalables, prenons le cas représentatif d'Adèle :

Tableau 3. Briser : interactions avec Adèle (semaine 1)

PE : Si je te dis : à la cantine, Sylvia a brisé un verre, pour toi ça veut dire quoi ?

Adèle : Ça veut dire que... elle [hésitation] elle... elle le ramasse.

PE : Elle le ramasse ? Pourquoi ?

Adèle : Parce que faut pas laisser par terre sinon y'a un enfant qui va marcher dessus et il... il va se piquer.

PE : Ah. Merci Adèle.

La paraphrase d'Adèle dans sa deuxième réplique révèle le scénario que l'élève se construit à partir de la situation qui lui est proposée. Bien que cette paraphrase contextuelle ne présente pas de synonyme, elle permet cependant de comprendre qu'Adèle a saisi l'acception de briser grâce à des termes tels " par terre », « se piquer » qui montrent que l'élève a compris que le verre était effectivement cassé.

Tableau 4. Hanter : interactions avec Adèle (semaine 1)

PE : Si je te dis : le château est hanté par des fantômes, pour toi ça veut dire quoi ?

Adèle : La peur.

PE : Ouais ? Le château est hanté par des fantômes. 
Adèle : Ben ça veut dire pour moi qu'il y a des fantômes.

PE : Ouais. Et...

Adèle : Ça fait peur.

PE : Et ça fait peur? OK.

Pour hanter, la réponse d'Adèle dans le cadre du protocole proposé ne permet pas d'être sûr de sa compréhension du verbe. En effet, ici la paraphrase « ça veut dire qu'il y a des fantômes » tient plus de la transformation syntaxique que de l'explication sémantique. En outre, l'allusion à la peur peut être attribuée à fantômes, que l'élève mobilise dans ses réponses, plutôt qu'au verbe de la phrase. Ce procédé ne signifie pas forcément que les élèves n'ont pas de représentation du sens des mots qui leur sont soumis, mais plutôt qu'ils ont alors du mal à verbaliser cette interprétation en faisant appel à leurs connaissances et au système lexical. Là se situe sans doute une difficulté de la paraphrase en maternelle, bien que, comme nous allons le voir, ce procédé va porter ses fruits par la suite.

Continuons à observer le cheminement d'Adèle durant la séance où lui sont proposés les verbes avec de nouveaux cooccurrents et dans une nouvelle acception (semaine 3) :

Tableau 5. Briser : interactions avec Adèle (semaine 3)

PE : D'accord. Si je te dis : la reine des neiges est triste car le prince lui a brisé le cœur ?

Adèle : Ça veut dire qu'elle est très triste.

PE : Oui ? Pourquoi?

Adèle : Et que... ben je sais pas trop.

PE : Parce que le prince lui a brisé le cœur ça veut dire quoi ?

Adèle : [INAUDIBLE]

Adèle : Ben il a brisé le cœur. Et... c'est... et ça peut nous rendre très triste.

PE : Ça veut dire quoi il lui a brisé le cœur ?

Adèle : Ça veut dire... ça veut dire qu'il est en mille morceaux.

PE : Il est en mille morceaux. D'accord.

Cette deuxième acception, de sens abstrait, semble difficile à appréhender par Adèle. L'élève, tout en parvenant à mobiliser les éléments cotextuels, fournit surtout une paraphrase ici proche de la transformation syntaxique qui reprend les termes de la phrase source : « il a brisé le cœur. Et... c'est... et ça peut nous rendre très triste ». Néanmoins, le pronom nous, ainsi que l'expression mille morceaux, font pressentir un scénario que l'enfant semble se construire, scénario qui semble proche de l'emploi métaphorique de briser. Une difficulté du même ordre se pose pour hanter:

Tableau 6. Hanter : interactions avec Adèle (semaine 3)

PE : Si je te dis... Luna est hantée par la peur à l'idée d'aller à la piscine car elle ne sait pas nager?

Adèle : Elle va se noyer et... [hésitation] elle peut mourir.

PE : Ouais ? Je te répète la phrase : Luna est hantée par la peur à l'idée d'aller à la piscine car elle ne sait pas nager? 


\section{Adèle : Elle va couler?}

Pour cette deuxième acception de hanter, l'élève s'est focalisée sur la deuxième partie de la phrase et il n'a pas été possible de savoir dans quelle mesure elle pouvait accéder ici au sens de hanter. Reste dans ses réponses le sentiment de peur et les conséquences de la situation proposée qu'on peut attribuer au contexte phrastique.

À travers cet exemple d'Adèle, nous voyons que les deux premières séances font émerger un savoir, parfois hésitant, à propos des deux verbes étudiés. Le recours à la paraphrase est permet ainsi d'avoir un premier aperçu de l'état des connaissances des élèves. Comme nous le pressentions, le sens abstrait est plus difficilement accessible. C'est d'ailleurs la raison pour laquelle nous avons cherché à travailler sur le rôle joué par les cooccurrents dans la précision du sens. Ces hésitations des élèves montrent pour nous l'intérêt d'étudier le verbe et ses entours avec les élèves. L'étude des cooccurrents par le jeu d'appariement aura-t-elle alors un impact sur les connaissances lexicales de ces élèves de grande section de maternelle?

Pour apporter des éléments de réponse à cette question, nous proposons un extrait du jeu d'appariement :

Tableau 7. Hanter : interactions durant le jeu d'appariement (semaine 5)

PE : Oui mais je demande à Antoine, si vous avez une idée vous la gardez dans votre tête et on la dira tout à l'heure. Alors on va utiliser quel mot ?

Antoine : Hanter... [hésitation]

PE : Oui, tu l'as dit.

Antoine : Hanter par la peur.

PE : Alors qui est hanté par la peur ?

Antoine : Le... [hésitation]

PE : Qui pourrait être hanté par la peur ?

Luna : Je sais, je sais.

PE : Mais tu as raison, on pourrait dire ça.

Antoine : Hum.

PE : Est-ce que c'est le fantôme qui est hanté par la peur ou est-ce que c'est quelqu'un d'autre?

Antoine : Quelqu'un d'autre.

$\mathbf{P E}$ : Alors on peut dire... on peut inventer un personnage, qui pourrait être hanté par la peur.

Luna : Je sais.

Antoine : Un monsieur.

PE : Un monsieur. Et hanté par la peur de quoi ?

Antoine : D'un fantôme.

PE : Oui d'en... de voir un fantôme peut-être?

Luna : Mais je sais...

PE : Luna? 
Luna : [hésitation] La maison est hantée de fantômes et le monsieur il a peur.

PE : La maison est hantée par des fantômes, ça c'est vrai. Et le monsieur a peur, c'est une autre idée. Adrien?

Adrien : C'est peut-être quelqu'un qui est déguisé en fantôme.

PE : Oui. Antoine, on le met où le mot hanter ? Oui Luna... Adèle ?

Adèle : En fait, peut-être... Un, un immeuble et dans toutes les... dans tous les étages... dans tous les étages partout dans l'immeuble, il y a des fantômes hantés.

PE : Est-ce que les fantômes ils sont hantés ? Non les fantômes ils...

Adèle : Non, non les... les gens sont hantés par la peur.

PE : De... ?

Adèle : Des fantômes. En plus y'en... y'en a dans tout l'appartement.

Cet extrait d'échange montre que les élèves sont maintenant capables de produire une réflexion plus poussée quant aux acceptions des mots étudiés. D'ailleurs, précédemment, lors d'un autre moment du jeu l'un d'entre eux regrette que "personne n'explique la technique », ce qui sera fait ensuite dans les justifications des appariements. Ainsi, le sens abstrait peut être mobilisé. Ici la forme de l'activité et les échanges avec les pairs profitent aux élèves. Ils s'essaient à la réflexion lexicale avec l'aide de l'enseignant, par exemple lorsque ce dernier demande si le fantôme est hanté par la peur, les élèves cherchent une explication à l'appui de leur réponse. Ils sont capables de proposer non seulement un scénario plausible (notamment Luna), mais aussi des paraphrases par équivalence fort judicieuses, comme Adèle : « Un, un immeuble et dans toutes les... dans tous les étages... dans tous les étages partout dans l'immeuble » pour gloser hanter. Ils progressent dans leur compréhension des unités lexicales. Petit à petit, les élèves sont plus attentifs au fonctionnement du lexique. En témoigne ce deuxième extrait du jeu d'appariement à propos de briser :

Tableau 8. Briser : interactions durant le jeu d'appariement (semaine 5)

\section{$[\ldots]$}

PE : Oui. Alors explique-moi Evan?

Evan : Eh ben il l'a bien brisé, du coup il est parti.

PE : Mais ça veut dire... est-ce que tu crois comme Hugo qu'il lui a planté une épée dans le cœur?

Plusieurs enfants : Non.

Evan : Mais non.

Enf. (non id.) : Un couteau.

PE : Un couteau ? D'accord, donc... et alors du coup elle est... elle est morte la princesse ?

Evan : Mais non. Je pense qu'il a fait peur avec un couteau, après il est reparti.

PE : Tout le monde est d'accord?

Plusieurs élèves : Non.

Hugo : On lui a vraiment brisé le cœur.

PE : Mais ça veut dire quoi alors, expliquez-moi ? 
Heyden : Ça veut dire que il est parti, il veut plus la revoir.

PE : Oui, mais est-ce que veut dire qu'il lui a vraiment... brisé le cœur en mille morceaux ?

Plusieurs élèves [de plus en plus fort $]$ : Non.

Hugo : Il est parti !

Cet extrait d'échange montre d'abord la difficulté de ces jeunes élèves à accéder au sens abstrait de briser dans l'expression briser le cour. Certains, comme Hugo, tentent de proposer un scénario qui leur semble plausible et qui réponde au mieux au synonyme de casser au sens concret de ne plus fonctionner. Ensuite, là aussi, la discussion et l'étayage permettent d'avancer dans la compréhension des variations sémantiques du verbe. La réponse d'Heyden est ici intéressante : après qu'un camarade a dit «il lui a vraiment brisé le cœur », il propose une paraphrase contextuelle : « ça veut dire qu'il est parti, il veut plus la revoir » et il peut dès lors permettre aux autres d'accéder à cette acception de briser, Hugo renchérit d'ailleurs : « il est parti !».

Ainsi, il apparait que de telles activités de paraphrasage mobilisant les cooccurrents favorisent la réflexion des tout jeunes élèves et leur permettent l'appréhension des différents sens de verbes étudiés. La réflexion dans ces extraits est poussée pour des enfants de 5 ans : à ce stade de l'acquisition, il est important de constater que les enfants explorent de la sorte les unités lexicales jusque dans leur sens métaphorique.

\subsection{Vers le réemploi des unités et de leurs cooccurrents}

En analysant les transcriptions des interactions lors des temps de synthèse (semaine 5), nous remarquons que les élèves réutilisent aussi les lexies étudiées. Le réemploi des unités avec leurs cooccurrents leur permet de mieux verbaliser leur interprétation du sens des unités proposées par leurs enseignants. Les élèves effectuent ces réemplois dans leurs gloses explicatives afin d'expliciter le sens qu'ils donnent, en fin de dispositif, aux différents mots étudiés. Ainsi pour les temps de synthèse :

Tableau 9. Briser : interactions durant la synthèse (semaine 5)

\section{$[\ldots]$}

Plusieurs enfants : Briser le cœur...

PE : À côté de briser le verre... donc quand on a brisé le verre ça voulait dire qu'on l'avait...

Enf. (non id.) : Cassé.

PE : Cassé en morceaux. Quand on a brisé le cœur, est-ce qu'on l'a cassé en mille morceaux ?

Evan : Non il... [Lève le doigt] on l'a cassé en deux.

PE : Tu es sûr?

Enf. (non id.) : Non. [Les enfants parlent en même temps [INAUDIBLE]

Evan : Il est brisé.

PE : Le cœur?

Heyden : Non c'est juste que... que... le prince il veut plus la revoir.

PE : Voilà, c'est juste que... elle est... la princesse est très malheureuse parce que le prince est parti, il lui a fait beaucoup de peine. D'accord ?

Evan : Et aussi il lui a fait du mal. 
PE : Mais son cœur n'est pas cassé.

Evan : Aussi il lui a fait du mal.

PE : Ben, du mal... de la peine en tous cas.

[...]

PE : Et là ? [Montrant une autre image]

Lilwen : Briser le cœur.

Evan : Il a brisé le cœur.

Heyden : Le prince est parti.

Evan : Le prince.

Heyden : Et il a fait de la peine à...

Evan : La princesse

PE : Voilà, il a fait de la peine à la princesse.

La dernière discussion ici reprend en partie les propos précédents, mais les enfants font preuve d'une capacité à préciser le sens de briser le cœur plus affirmée que précédemment. Ils sont capables de paraphraser en reprenant les termes, tout en proposant une explication contextuelle : Heyden rappelle que « le prince est parti », il répète les mots de l'enseignante : « il a fait de la peine », au moment où Evan réemploie l'expression briser le cœur; à eux deux, ces jeunes élèves formulent une sorte de définition contextuelle par leur paraphrase. Evan, qui avait précédemment des difficultés à accéder au sens abstrait de ce verbe, parait réemployer l'expression briser le cœur pour mieux se l'approprier ici dans le cadre de l'échange conduit pour l'enseignante qui insiste d'ailleurs sur le fait que la douleur n'est pas forcément physique lorsqu'elle dit à Evan : « ben, du mal... de la peine en tout cas ». C'est d'ailleurs la paraphrase de cette-dernière qui fournit un élément de précision du sens : «il lui a fait beaucoup de peine », terme repris en fin d'extrait par les élèves. Pour des expressions semi-figées telles briser le cœur, c'est l'environnement phrastique (ici la princesse était triste dans la phrase proposée en séance précédente) qui est mobilisé pour parvenir à des paraphrases judicieuses réemployant les mots étudiés, en les recontextualisant. Paraphrase des élèves et de l'enseignante se mêlent ainsi pour faire avancer la réflexion lexicale, tout en mobilisant les unités étudiées et leur cotexte.

Le réemploi est également manifeste pour le verbe hanter:

Tableau 10. Hanter : interactions durant la synthèse (semaine 5)

PE : Chut. Claudie, selon toi, est-ce que tu prendrais ?

Claudie : Hanté par la peur.

PE : Alors elle est hantée par la peur... de quoi ?

Plusieurs enfants : D'un loup / de le loup.

Claudie : De... de.... du papillon.

Pharell : Du papillon ? ça... est-ce que ça fait peur un papillon.

Jordan : [se couche à moitié sur la table] D'un loup...

PE : Ah ben dis donc oui c'est... C'est original hein. Qu'est-ce qu'il pourrait faire le papillon, soyons précis.

Claudie : Ah oui parce qu'elle regarde le papillon elle a... elle s'enfuit. 
PE : Elle s'est enfuie.

Pharell : Parce que ça la surprend ? Peut-être ça la surprend ? Ou peut-être c'est... on le voit pas mais y'a un méchant loup là ? [Claudie essaie de poser la carte sur un emplacement de la feuille]. Elle est hantée par la peur de... de voir un loup.

PE : De voir un loup peut-être.

Pharell : Elle est hantée par la peur de voir un loup du coup ben... dans ses... dans ses yeux elle voit un loup.

L'extrait ci-dessus montre les progrès des élèves pour l'acception psychique de hanter. L'association du verbe avec son complément est rapidement proposée par Claudie, et complétée par Pharell. Si Claudie hésite quant au scénario et ne peut aller jusqu'au bout de sa pensée, par l'emploi du verbe s'enfuir, il semble cependant qu'elle ait construit une représentation cohérente du sens ici de hanter. Pharell quant à lui réemploie et prolonge le verbe et son complément : « hantée par la peur du loup ». Cette paraphrase nous parait montrer là les progrès effectués, car ici un nouveau contexte est judicieusement proposé par l'élève : il ne s'agit plus de la peur des fantômes, mais de celle du loup. L'élève réemploie les termes dans une nouvelle situation, via la paraphrase. Pharell donne ici l'exemple de la « combinaison entre une partie invariante que le locuteur reprend à un énoncé source (= ES), point de départ du processus de reformulation, et une partie variante, nouvelle, que le locuteur articule à la partie invariante pour produire un énoncé reformulé (=ER)» (Martinot, 2010, p. 64). De la sorte, le dispositif proposé semble avoir permis à ces élèves de grande section de maternelle d'explorer le fonctionnement du lexique et de ses unités pour pouvoir s'en saisir au point de tenter des réemplois via les paraphrases lors des discussions.

\section{Conclusion}

Malgré les limites relatives aux difficultés liées à la reformulation de phrases et termes de sens abstrait avec de jeunes apprenants, ou le nombre restreint ici d'enfants concernés, cette étude montre néanmoins que sens et syntagme, via la paraphrase et le cotexte sont favorables aux acquisitions dès l'école maternelle. Compétence lexicale et compétence paraphrastique semblent indissociables. Dès lors il parait possible de proposer tôt dans la scolarité des activités de réflexion autour des mots, notamment des verbes, en faisant appel à la capacité des élèves à interroger les unités lexicales, et à développer leur « sensibilité lexicale » (Tremblay, 2017, p. 4). De la sorte, l'enseignant pourra donner à ses jeunes élèves le gout de l'exploration et, par là, des pistes pour produire l'inférence lexicale, structurer et accroitre leur lexique. Ces résultats encouragent à proposer aux élèves, dès la maternelle, des jeux de réflexion sur la langue, parfois très ciblés, pour leur permettre de mieux appréhender le fonctionnement de notre système linguistique et de développer leur curiosité lexicale.

\section{Références bibliographiques}

Aurnague, M. \& Garcia-Debanc, C. (2016). Les verbes de déplacement comme contenu d'enseignement du lexique à l'école primaire : modélisation sémantique et analyse de productions d'élèves. Actes du $5^{e}$ Congrès Mondial de Linguistique Française, Tours, 4-8 juillet 2016, Université François Rabelais (France). https://www.shsconferences.org/articles/shsconf/abs/2016/05/shsconf cmlf2016 07001/shsconf cmlf2016 07001.html

Babin, J.-P. (2000). Lexique mental et morphologie lexicale. Berne : Peter Lang.

Bassano, D. (2005). Production naturelle précoce et acquisition du langage : l'exemple du développement des noms. Lidil 31, 61-84.

Bassano, D. (2010). L'acquisition des verbes en français : un exemple de l'interface lexique / grammaire. Synergies France 6, 27-39 
Chanfrault-Duchet, M.-F. (2004). Vers une approche syntagmatique du lexique en didactique du français. Dans É. Calaque et J. David (dir.). Didactique du lexique. Contextes, démarches, supports. Bruxelles : De Boeck, 103114.

Dubois, J. \& Dubois-Charlier, F. (1997). Les verbes français. https://talep.lislab.fr/FondamenTAL/Ouvrage_LVF.pdf (dernère consultation de 3 mai 2020).

Florin, A. (2010). Le développement du lexique et l'aide aux apprentissages. ERES, Enfances \& Psy 47, 30-41.

Garcia-Debanc, C., Duvignau, K., Dutrait, C., Gangneux, M. (2009). Enseignement du lexique et production écrite. Un travail sur les verbes de déplacement à la fin de l'école primaire. Pratiques 141-142, 141-142.

Martinot, C. (2010). Reformulation et acquisition de la complexité linguistique. Travaux de linguistique 61, 63-96.

Picoche, J. (1999). Dialogue autour de l'enseignement du vocabulaire. Études de Linguistique Appliquée, 116, 325350.

Picoche, J. (2011). Lexique et vocabulaire : quelques principes d'enseignement à l'école. Eduscol, https://cache.media.eduscol.education.fr/file/Dossier_vocabulaire/14/4/Jacqueline Picoche 111202 avec couv 201144.pdf.

Polguère A., Tremblay O. (2003) Qu'y a-t-il à l'intérieur de NOIX ? Ou comment décortiquer les unités lexicales. Dans J. David \& F. Grossmann, La Lettre de l'AIRDF 33. Dossier : Enseigner-apprendre le lexique, 27-30.

Polguère, A. (2002). Notions de base en lexicologie. Montréal : Observatoire de Linguistique Sens-Texte.

Roubaud, M.-N. \& Moussu, M.-J. (2012). Un enseignement structuré du lexique dès la maternelle au service de l'écriture. Pratiques 155-156, 109-126.

Roubaud, M.-N. \& Moussu, M.-J. (2014). Favoriser l'acquisition lexicale chez de jeunes enfants : « le dire par le faire ». Dans C. Garcia-Debanc, C. Masseron, C. Ronveaux, Enseigner le lexique. Namur : presses universitaires de Namur, 85-101.

Sardier, A \& Grossmann, F. (2010). Comment favoriser le réemploi lexical ? Recherches 53, 9-34.

Sardier, A. (2016a). Enseignement-apprentissage du lexique: vers le réemploi lexical en grande section de maternelle. Repères 54, 207-232.

Sardier, A. (2016b). Effets de la prise en compte de la dimension syntagmatique du lexique sur le développement de la compétence lexicale en début de collège. Actes du $5^{e}$ Congrès Mondial de Linguistique Française, Tours, 4-8 juillet 2016, Université François Rabelais (France). conferences.org/articles/shsconf/abs/2016/05/shsconf cmlf2016 07016/shsconf cmlf2016 07016.html

Sardier, A. (2018). Inciter les élèves à recourir au cotexte pour inférer le sens des unités lexicales. Dans C. Ronveaux \& O. Tremblay, La Lettre de l'AIRDF 64. Dossier : La didactique du lexique, 36-42.

Tremblay, O. (2009). Une ontologie des savoirs lexicologiques pour l'élaboration d'un module de cours en didactique du lexique. Thèse de doctorat soutenue à l'université de Montréal : Département de didactique, Faculté des sciences de l'éducation.

Tremblay, O. (2017). Entrer dans le lexique par la porte des sentiments. Correspondance 6, http://correspo.ccdmd.qc.ca/index.php/document/entrer-dans-le-lexique-par-la-porte-des-sentiments/

Tsedryk, A. (2014). Un autre regard sur l'apprenant avancé : sa capacité de paraphraser. Actes du $4^{e}$ Congrès Mondial de Linguistique Française, Berlin, 19-23 juillet 2014, Université de Berlin (Allemagne). https://www.shsconferences.org/articles/shsconf/abs/2014/05/shsconf_cmlf14_01134/shsconf_cmlf14_01134.html 


\section{Annexes}

Annexe 1 Affiche collective mentionnant les premières acceptions pour briser et hanter

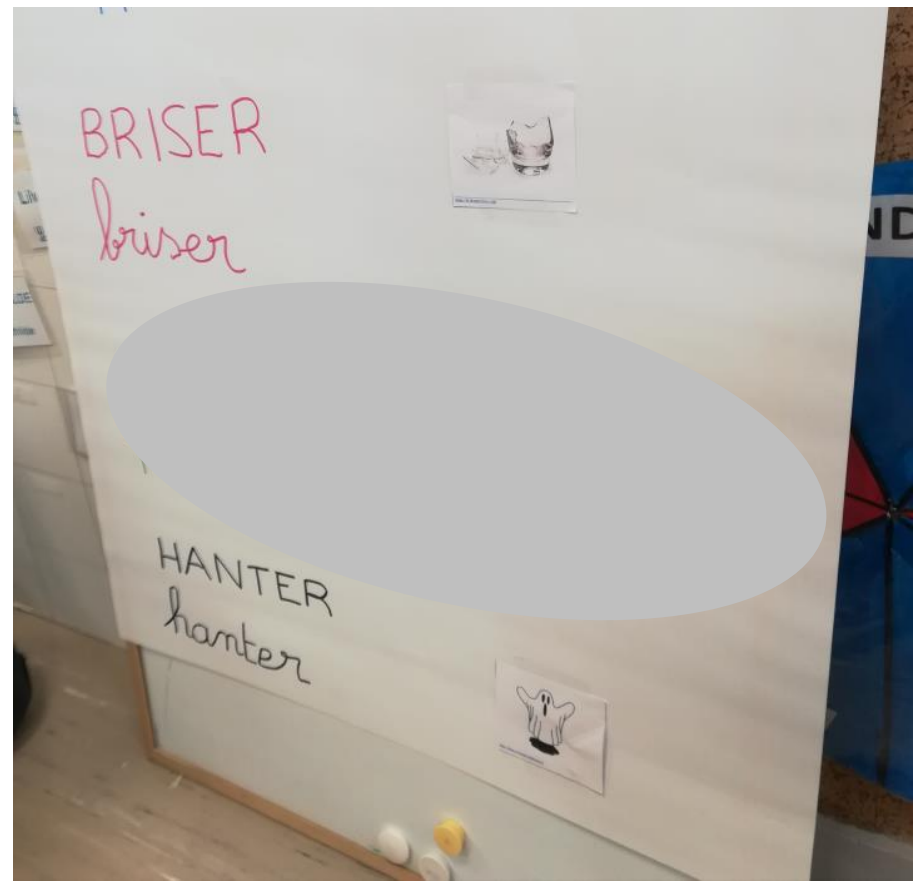

Annexe 2 Illustration du jeu d'appariement

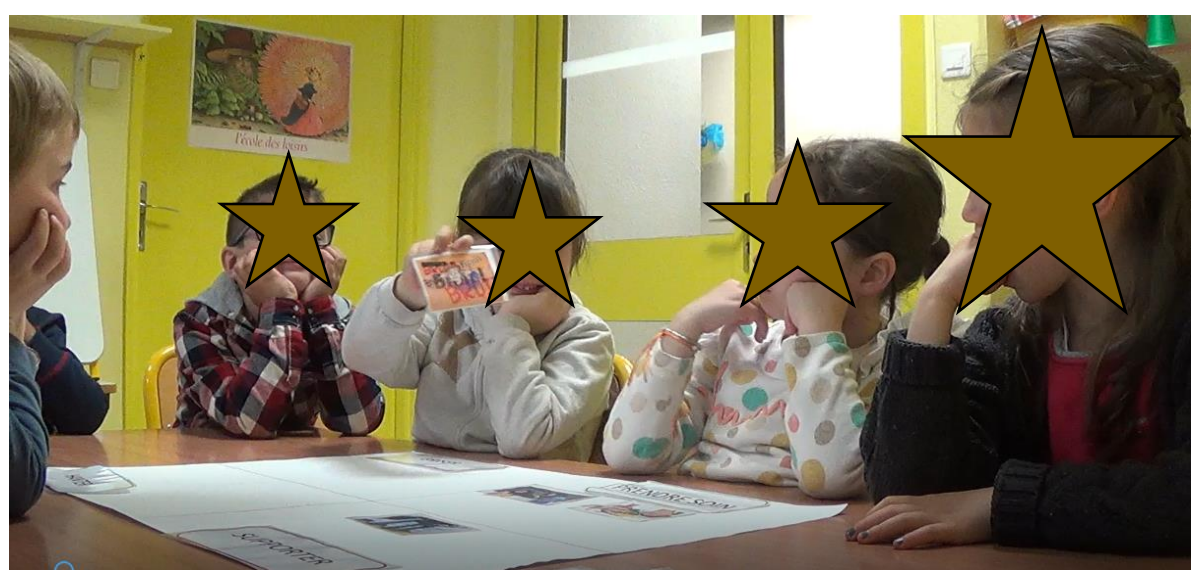


' Ces cooccurrents fréquents sont à présent assez aisément identifiables grâce à des logiciels tel Antidote et son dictionnaire des cooccurrences, ou grâce à l'onglet "concordance" sur le portail lexical du Centre e Ressources Textuelles et Lexical (CNRTL).

ii Le verbe supporter et la construction à verbe support prendre soin ont aussi été choisis par les enseignants, mais nous n'en traitons pas ici l'analyse.

iii Par exemple dans les albums de Mies Van Hout, qui ont été lus durant la phase d'imprégnation. 